\title{
CLASIFICACIÓN DE ESTUDIANTES DE ENFERMERÍA MEXICANOS ACORDE CON UN INDICADOR DE ESTILO DE VIDA SALUDABLE
}

\author{
CLASSIFICATION OF MEXICAN NURSING STUDENTS ACCORDING \\ TO A HEALTHY LIFESTYLE INDEX
}

\section{CLASSIFICAÇÃO DE ESTUDANTES MEXICANOS DE ENFERMAGEM CONFORME UM INDICADOR DE ESTILO DE VIDA SAUDÁVEL}

\author{
Cleotilde García* \\ ViCENTA GÓMEZ** \\ Matiana Morales*** \\ LORENA ChAPARRo-DíAZ ${ }^{* * * *}$ \\ Sonia CARreño-Moreno ${ }^{* * * * *}$
}

\begin{abstract}
RESUMEN
Objetivo: Describir agrupaciones de estudiantes de Enfermería acorde con un indicador de estilo de vida saludable. Material y método: Estudio cuantitativo exploratorio realizado en la Universidad Autónoma del Estado de México. La muestra estuvo constituida por 865 estudiantes de Enfermería. Se realizó un análisis clúster no jerárquico por $k$-means con una partición en 3 agrupaciones, las cuales representan el indicador de Estilo de vida. Resultados: Se distinguieron 3 agrupaciones que representan el indicador de estilo de vida de los estudiantes: Estilo de vida poco saludable $(\mathrm{n}=392)$, saludable $(\mathrm{N}=402)$ y muy saludable $(\mathrm{n}=71)$. Las dimensiones de estrés, consumo de tabaco y alcohol son las conductas de riesgo más frecuentes en los estudiantes. Conclusión: Un gran número de estudiantes de Enfermería se agruparon en el clúster de estilo de vida poco saludable. Se requiere fortalecer acciones de promoción de estilos de vida saludable en entornos universitarios.
\end{abstract}

Palabras clave: Estilo de vida; Estudiantes de Enfermería; Educación en enfermería; Universidades.

\section{ABSTRACT}

Objective: To describe groups of Nursing students according to a healthy lifestyle index. Material and method: Exploratory and quantitative study, conducted at the Universidad Autónoma del Estado de México (UAEM).

\footnotetext{
*Enfermera. Profesora Facultad de Enfermería y Obstetricia, Universidad Autónoma del Estado de México. Toluca, México. Email: cgarc0506@yahoo.com

**Enfermera. Profesora Facultad de Enfermería y Obstetricia, Universidad Autónoma del Estado de México. Toluca, México. Email:vgm2259@hotmail.com

***Enfermera. Profesora Facultad de Enfermería y Obstetricia, Universidad Autónoma del Estado de México. Toluca, México. Email: maty_2402@yahoo.com.br

****Enfermera. Profesora Universidad Nacional de Colombia - Sede Bogotá - Facultad de Enfermería, Bogotá, Colombia. Email: olchaparrod@unal.edu.co. Autor de correspondencia

*****Enfermera. Profesora Universidad Nacional de Colombia - Sede Bogotá - Facultad de Enfermería, Bogotá, Colombia. Email: spcarrenom@unal.edu.co
} 
The sample consisted of 865 Nursing students. A non-hierarchical cluster analysis was made using k-means with a partition in three groups, which represent the lifestyle index. Results: Three clusters representing the student's lifestyle index were distinguished: poor healthy lifestyle $(n=392)$, healthy lifestyle $(n=402)$ and very healthy lifestyle $(n=71)$. Stress, smoking and drinking, are the most common risk behaviours among students. Conclusion: A large number of Nursing students were grouped in the poor healthy lifestyle cluster. Therefore, it is necessary to promote healthy lifestyles in university settings.

Key words: Life Style; Students; Education; Nursing; Universities.

\section{RESUMO}

Objetivo: Descrever agrupamentos de estudantes de Enfermagem conforme um indicador estilo de vida saudável. Material e método: Estudo quantitativo exploratório, realizado na Universidade Autônoma do Estado do México (UAEM). A amostra foi constituída por 865 estudantes de Enfermagem. Realizou-se uma análise de cluster não hierárquica de agrupamento utilizando k-means com uma partição em três grupos, os quais representam o indicador de estilo de vida. Resultados: Foram distinguidos três grupos que representam o indicador de estilo de vida dos estudantes: estilo de vida pouco saudável ( $n=392)$, estilo de vida saudável $(\mathrm{n}=402)$ e estilo de vida muito saudável $(\mathrm{n}=71)$. As dimensões de estresse, consumo de tabaco e álcool, são as condutas de risco mais frequentes dos estudantes. Conclusão: Um grande número de estudantes de Enfermagem foi agrupado no cluster de estilo de vida pouco saudável. É preciso fortalecer açóes de promoção de estilos de vida saudáveis no ambiente universitário.

Palavras chave: Estilo de vida; Estudantes; Educação; Enfermagem; Universidades.

Fecha recepción: 17/10/2018

Fecha aceptación: 14/03/2019

\section{INTRODUCCIÓN}

El estilo de vida de una persona se constituye en las costumbres y comportamientos que caracterizan su forma de vivir, influyendo factores psicológicos, sociales, culturales y económicos que afectan directamente la salud ${ }^{(1,2)}$. La persona puede establecer prácticas saludables y no saludables que van interactuando entre $s^{\prime(3)}$; sin embargo, cuando prevalecen las prácticas poco saludables existe mayor probabilidad de padecer enfermedades crónicas.

La Organización Mundial de la Salud (OMS) ${ }^{(4)}$ menciona que las principales causas de la aparición de las enfermedades crónicas no transmisibles (ECNT) son los comportamientos mal sanos como consumo de tabaco y alcohol, inactividad física y dietas inadecuadas, todos ellos relacionados con el estilo de vida de la persona. En México, por ejemplo, se han señalado como importantes factores de riesgo de padecer enfermedades crónicas debido al sobrepeso, obesidad y falta de actividad física ${ }^{(5)}$.

El panorama mundial denota un incremento en las conductas sedentarias, incluso en los estudiantes universitarios $^{(6)}$; sehan reportado cifras preocupantes sobre los hábitos de vida de los estudiantes: solo el 13,6\% de los jóvenes universitarios tienen una alimentación adecuada y el otro gran segmento presenta dietas con alto consumo en colesterol y sal, o la ingesta de una sola comida al día ${ }^{(7-9)}$. En Inglaterra, el $70 \%$ de los estudiantes no realizan ejercicio y el 56\% reconoce un alto consumo de alcohol $^{(7)}$; en México se encuentran cifras por encima del $50 \%$ en el uso de cigarrillo, fumando hasta 10 en el día ${ }^{(1)}$.

Esta situación representa un importante problema de salud pública, pues implica un riesgo a mediano y corto plazo de personas que padecerán ECNT, debido a la falta de estilos de vida saludable; la presencia de obesidad y sobrepeso, especialmente, se debe a esta falta de buenos hábitos alimentarios y de conductas deportivas ${ }^{(10-12)}$. Este contexto pone a prueba a las universidades, para que adopten políticas que promuevan los estilos y comportamientos de vida saludables.

Considerando el reporte de inadecuados estilos de vida en estudiantes universitarios, además de que 
se tienen reportes de frecuencias absolutas de los mismos acorde con comportamientos particulares tales como el consumo de alcohol o cigarrillo, mala alimentación y sedentarismo, se requiere conocer un indicador global de estilo de vida que agrupe los comportamientos, que dé cuenta de manera general acerca del estilo de vida, además de conocer cómo se agrupan estudiantes universitarios acorde con dicho indicador.

Teniendo en cuenta el panorama anterior, el objetivo de este estudio fue describir agrupaciones de estudiantes de Enfermería acorde con un indicador de estilo de vida saludable.

\section{MATERIAL Y MÉTODO}

Tipo de estudio: Estudio descriptivo y exploratorio que se desarrolló en las siguientes fases:

1. Descripción de los estilos de vida saludable en las dimensiones de descanso y sueño, actividad física, estrés, consumo de tabaco, consumo de alcohol y alimentación.

2. Construcción de un indicador de estilo de vida saludable.

3. Análisis clúster no jerárquico k-means para determinar la agrupación de los estudiantes, de acuerdo con el indicador de estilo de vida saludable.

Muestra: La muestra estuvo constituida por 865 estudiantes de Enfermería de segundo, cuarto, sexto y octavo semestre, de la Universidad Autónoma del Estado de México, los cuales representan el 67\% de los estudiantes de la carrera. El tamaño de muestra fue calculado con una confianza del $95 \%$ y un error máximo admisible del 5\%. Fueron considerados criterios de inclusión estar matriculado en la carrera de Enfermería y estar cursando la asignatura obligatoria de cada semestre de acuerdo con el plan curricular de estudios. Los estudiantes fueron reclutados en la Facultad de Enfermería por las investigadoras, quienes administraron el cuestionario ESVISAUN en los salones de clase, en el segundo semestre de 2017. Las personas incluidas en este estudio participaron de manera voluntaria y anónima previo consentimiento informado. Los datos fueron custodiados por los investigadores y los nombres fueron reemplazados por códigos alfanuméricos para preservar la confidencialidad.
Instrumento: Los datos fueron recolectados con el cuestionado ESVISAUN versión 3 el cual valora los estilos de vida saludable y salud en estudiantes universitarios. El cuestionario tiene 92 preguntas acerca del peso, actividad física, sueño, estrés, consumo de tabaco, consumo de alcohol y alimentación; su escala de respuestas es categórica y tipo Likert. Las preguntas que se contestan de manera categórica-dicotómica si/no tienen que ver con el consumo de alcohol y cigarrillo, mientras que las que tienen escala Likert valoran autopercepción de la frecuencia de hábitos no saludables y va desde muy nunca, casi nunca, a veces, a menudo y muy a menudo, con puntuaciones de cero a cinco ${ }^{(13)}$.

Construcción del indicador: La construcción del indicador para evaluar el estilo de vida de los estudiantes de Enfermería que participaron en esta investigación tuvo en cuenta las 6 dimensiones del ESVISAUN. Se le asignó una ponderación o peso a cada dimensión del cuestionario, esto, con base a la revisión de literatura realizada previamente por las autoras. El proceso para la elaboración del indicador fue de la siguiente forma:

1. Establecimiento de la ponderación de cada una de las preguntas dentro de cada dimensión del cuestionario, dando el mismo peso a cada una de las preguntas dentro de su dimensión.

2. Establecimiento de la ponderación de cada una de las dimensiones. La ponderación se realizó dando un peso porcentual a cada una de las dimensiones: Descanso y sueño: $20 \%$, actividad física $20 \%$, estrés $20 \%$, consumo de sustancias $20 \%$ (tabaco 10\%, alcohol 10\%) y alimentación 20\%. La decisión de hacer una ponderación equivalente para todas las dimensiones se basó en que la literatura no reporta algún estilo de vida más relevante que otro, sino que en conjunto son importantes.

3. Establecimiento de un indicador de estilo de vida como la suma ponderada de los puntajes obtenidos de cada una de las dimensiones del cuestionario.

4. El Cuadro 1 indica el peso que aporta cada dimensión para el indicador de estilo de vida, así como la puntuación del indicador propuesto.

Las autoras consideraron, a partir del análisis estadístico realizado, que los estudiantes podían dividirse en tres grandes grupos: estudiantes 
cuyo estilo de vida es poco saludable, estudiantes con un estilo de vida saludable y los estudiantes cuyo estilo de vida es muy saludable. De acuerdo con los resultados obtenidos del indicador de estilo de vida (Gráfico 1), se evidencia que la observación realizada por las autoras sobre las tres agrupaciones es razonable ya que aunque se muestra una distribución con la mayoría de los datos en cuatro grupos, el conjunto de datos que se incorporan en el último grupo de la gráfica, es decir un pequeńo grupo que tiene un muy buen estilo de vida, no evidencia relevancia estadística, ni teórica, puesto que la decisión de cuatro grupos implicaría denominar dos pequeños grupos que en general tienen estilo de vida muy saludable. Por lo anterior, la decisión fue reportar tres agrupaciones por la similitud en su indicador de estilo de vida y por la categorización o denominación que podría asignarse teóricamente.

Cuadro 1. Peso de cada sección dentro del indicador de estilo de vida.

\begin{tabular}{|c|c|c|c|c|}
\hline Sección & Peso & Puntuación & Min. & Máx. \\
\hline Descanso y sueño & $20 \%$ & $\begin{array}{l}\text { Valores mayores a } 0 \text {. Puntuaciones altas sugieren que el } \\
\text { estudiante descansa más }\end{array}$ & 1,2 & 5,2 \\
\hline Actividad física & $20 \%$ & $\begin{array}{l}\text { Valores mayores o iguales a } 0 \text {. Puntuaciones altas sugieren } \\
\text { que el estudiante realiza mucha actividad física }\end{array}$ & 0,0 & 25,8 \\
\hline Estrés & $20 \%$ & $\begin{array}{l}\text { Valores entre } 0 \text { y } 2.8 \text {. Altas puntuaciones sugieren bajos } \\
\text { niveles de estrés }\end{array}$ & 0,3 & 2,6 \\
\hline Consumo de tabaco & $10 \%$ & $\begin{array}{l}\text { Valores mayores o iguales a } 0 \text {. Altas puntuaciones sugieren } \\
\text { un menor consumo de tabaco }\end{array}$ & 0,0 & 4,0 \\
\hline Consumo de alcohol & $10 \%$ & $\begin{array}{l}\text { Valores mayores o iguales a } 0 \text {. Altas puntuaciones sugieren } \\
\text { un menor consumo de alcohol }\end{array}$ & 0,0 & 5,0 \\
\hline Alimentación & $20 \%$ & $\begin{array}{l}\text { Valores entre } 0.3 \text { y } 2.9 \text {. Altas puntuaciones una mejor } \\
\text { alimentación }\end{array}$ & 0,5 & 2,6 \\
\hline Estilo de vida & $100 \%$ & $\begin{array}{l}\text { Valores mayores o iguales a } 0.3 \text {. Altas puntuaciones en el } \\
\text { indicador sugieren un mejor estilo de vida }\end{array}$ & 1,1 & 7,6 \\
\hline
\end{tabular}

Gráfico 1. Histograma agrupación por k-means.

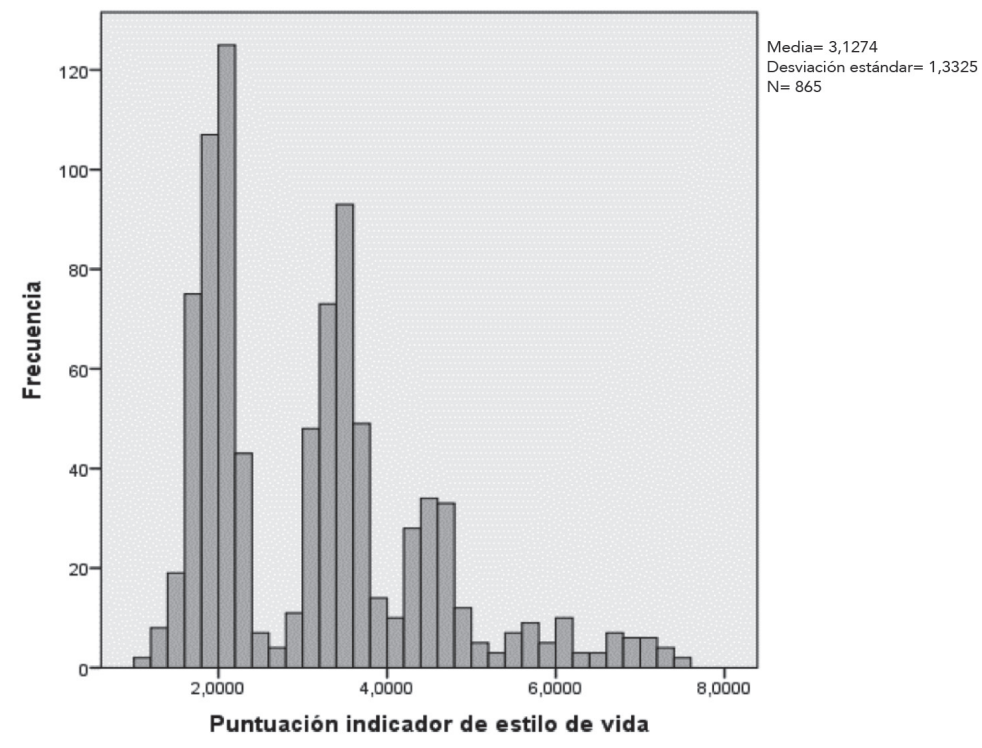


Análisis estadístico: Se realizó un análisis clúster no jerárquico por $k$-means, utilizando la medida de similitud de distancia Euclidiana, con una partición en 3 agrupaciones, las cuales representan el indicador de Estilo de vida. La razón de su utilización radica en la misma manera en la que el procedimiento se lleva a cabo ${ }^{(14)}$ : Particionar los individuos en $\mathrm{k}$ grupos; asignar un individuo al grupo cuyo centroide (media) sea el más cercano: recalcular el centroide para el grupo que recibe el nuevo individuo y para el grupo que perdió al individuo; repetir el paso anterior hasta que no se realicen más reasignaciones.

Para los análisis correspondientes al estudio se utilizó el software SPSS versión 23.

\section{RESULTADOS}

Las edades de los estudiantes que participaron en el estudio oscilaron entre los 18 y 46 años; en su mayoría fueron mujeres de estado civil soltera.

\section{Análisis clúster}

Mediante el análisis clúster se distinguieron tres agrupaciones resultantes de la partición que se realizó por el k-means; los grupos quedaron conformados así, clúster 1: 392 estudiantes, clúster 2: 402 estudiantes y clúster 3: 71 estudiantes. Dado que valores altos del indicador se refieren a un mejor estilo de vida, la Tabla 1 indica que el grupo 1 corresponde a los estudiantes con peor estilo de vida, el grupo 2 estudiantes con estilo de vida saludable y grupo 3 estudiantes estilo de vida muy saludable, compuesto por solo 71 de ellos.

En la Tabla 2 se presentan las características de los estudiantes, según su distribución por clúster. En los clústeres 1 y 2 la frecuencia de mujeres supera el $80 \%$, a diferencia del clúster 3, donde el porcentaje femenino $(52,1 \%)$ y masculino $(47,9 \%)$ no tuvo una diferencia importante. Respecto al estado civil de los estudiantes, se observa una marcada tendencia al estado civil soltero en los tres clústeres con más del $87 \%$ de los participantes en cada grupo, especialmente en el grupo 3 (97,2\%); seguido del estado civil unión libre y casado.

Tabla 1. Resumen de los clúster del indicador de estilo de vida.

\begin{tabular}{lccccccc}
\hline \multicolumn{1}{c}{ Clúster } & Media & DE & Mediana & Mín & Máx & Q1 & Q3 \\
\hline Poco saludable $(\mathrm{n}=392)$ & 1,955 & 0,259 & 1,97 & 1,115 & 2,84 & 1,78 & 2,12 \\
Saludable $(\mathrm{n}=402)$ & 3,54 & 0,548 & 3,54 & 2,885 & 4,955 & 3,329 & 4,245 \\
Muy saludable $(\mathrm{n}=71)$ & $\mathbf{6 , 1 9 4}$ & 0,709 & 6,105 & 4,98 & 7,56 & 5,625 & 6,8 \\
\hline
\end{tabular}

Tabla 2. Características de los estudiantes según clúster en que se agruparon.

\begin{tabular}{cccccccc}
\hline \multirow{2}{*}{ Característica } & & $\begin{array}{c}\text { Clúster 1 } \\
\text { Frecuencia }\end{array}$ & Porcentaje & $\begin{array}{c}\text { Clúster 2 } \\
\text { Frecuencia }\end{array}$ & Porcentaje & $\begin{array}{c}\text { Clúster 3 } \\
\text { Frecuencia }\end{array}$ & Porcentaje \\
\hline \multirow{2}{*}{ Sexo } & Femenino & 355 & 90,6 & 331 & 82,3 & 37 & 52,1 \\
& Masculino & 37 & 9,4 & 71 & 17,7 & 34 & 47,9 \\
\hline \multirow{2}{*}{ Estado civil } & Soltero & 344 & 87,8 & 356 & 88,6 & 69 & 97,2 \\
& Casado & 19 & 4,8 & 19 & 4,7 & 1 & 1,4 \\
& Viudo & 2 & 0,5 & 1 & 0,2 & 0 & - \\
& Unión & 23 & 5,9 & 23 & 5,7 & 1 & 1,4 \\
& libre & & & & & & \\
\hline & Separado & 4 & 1 & 3 & 0,7 & 0 & - \\
\hline
\end{tabular}




\section{Descripción de los estilos de vida}

En la Tabla 3 se muestra el análisis descriptivo de las seis dimensiones que componen el indicador de estilo de vida.

La dimensión de estrés (1,364 DS 0,414), consumo de tabaco $(3,614$ DS 0,876$)$ y consumo de alcohol (3, 614 DS 0,876) indican una presencia alta en los hábitos de vida de los estudiantes, siendo este un factor de riesgo importante en la tendencia a estilos de vida poco saludables, así como la falta de descanso y sueño $(3,144$ DS 0,660) adecuados, la poca e ineficiente actividad física $(5,807 \mathrm{DS}$ $6,478)$ y los hábitos alimentarios (1,541 DS 0,358) malsanos.

\section{Descripción de las dimensiones entre agrupa- ciones del indicador de estilos de vida}

La dimensión de descanso y sueño expresa valores que varían poco entre cada clúster. Sin embargo, se evidencia que la media de la dimensión aumenta a medida que el indicador expresa mejores estilos de vida, desde poco saludable (3,07 DS 0,64), saludable $(3,16$ DS 0,65$)$ y muy saludable $(3,38$ DS 0,7) (Tablas 4, 5 y 6).
La dimensión actividad física expresó un aumento llamativo entre las agrupaciones, coherente con la cualidad poco, saludable o muy saludable de cada clúster. El aumento de la media de esta dimensión va desde poco saludable $(0,16 \mathrm{DS}$ $0,75)$, saludable $(8,66$ DS 2,78$)$ y muy saludable (20,81 DS 4,06) (Tablas 4, 5 y 6).

La dimensión estrés, al igual que la dimensión descanso y sueńo, expresa valores con pocas variaciones, pero que aumentan de manera coherente entre cada agrupación. El aumento de los valores va desde poco saludable (1,31 DS 0,41), saludable $(1,37$ DS 0,4$)$ y muy saludable $(1,5$ DS $0,42)$. Además, se observan desviaciones estándar muy similares entre los clústeres (Tablas 4, 5 y 6).

Frente al consumo de tabaco se observaron variaciones pequeñas en los valores entre cada agrupación, además de no expresar un patrón de variación particular. Es así como la agrupación denominada como saludable $(3,66$ DS 0,82) expresó menor consumo de tabaco que la agrupación muy saludable $(3,44$ DS 0,96$)$ y poco saludable $(3,58$ DS 0,9$)$. De cualquier forma, la agrupación poco saludable expresó el mayor consumo de tabaco (Tablas 4, 5 y 6).

Tabla 3. Resumen de las puntuaciones de las dimensiones.

\begin{tabular}{lccc}
\hline & Media & DE & Asimetría \\
\hline Descanso y sueño & 3,144 & 0,66 & 0,112 \\
Actividad física & 5,807 & 6,478 & 1,116 \\
Estrés & 1,364 & 0,414 & 0,414 \\
Consumo de tabaco & 3,614 & 0,876 & $-2,129$ \\
Consumo de alcohol & 3,614 & 0,876 & $-2,129$ \\
Alimentación & 1,541 & 0,358 & 0,258 \\
Estilo de vida & 3,127 & 1,333 & 0,981 \\
\hline
\end{tabular}

Tabla 4. Resumen puntuación estilos de vida poco saludables.

\begin{tabular}{lccc}
\hline & Media & DE & Asimetría \\
\hline Descanso y sueño & 3,07 & 0,64 & 0,1 \\
Actividad física & 0,16 & 0,75 & 8,38 \\
Estrés & 1,32 & 0,41 & $-0,12$ \\
Consumo de tabaco & 3,58 & 0,9 & $-2,11$ \\
Consumo de alcohol & 3,94 & 1,14 & $-6,07$ \\
Alimentación & 1,44 & 0,34 & 0,26 \\
Estilo de vida & 1,95 & 0,25 & $-0,143$ \\
\hline
\end{tabular}


Tabla 5. Resumen puntuación estilos de vida saludables

\begin{tabular}{lccc}
\hline & Media & DE & Asimetría \\
\hline Descanso y sueño & 3,16 & 0,65 & 0,03 \\
Actividad física & 8,66 & 2,78 & 0,89 \\
Estrés & 1,37 & 0,4 & $-0,5$ \\
Consumo de tabaco & 3,66 & 0,82 & $-2,32$ \\
Consumo de alcohol & 4,01 & 1,08 & $-0,53$ \\
Alimentación & 1,6 & 0,33 & 0,29 \\
Estilo de vida & 3,72 & 0,54 & 0,69 \\
\hline
\end{tabular}

Tabla 6. Resumen puntuación estilos de vida muy saludables.

\begin{tabular}{lccc}
\hline & Media & DE & Asimetría \\
\hline Descanso y sueño & 3,38 & 0,7 & 0,32 \\
Actividad física & 20,81 & 4,06 & $-0,26$ \\
Estrés & 1,5 & 0,42 & $-0,13$ \\
Consumo de tabaco & 3,44 & 0,96 & $-1,43$ \\
Consumo de alcohol & 3,6 & 1,15 & $-0,2$ \\
Alimentación & 1,74 & 0,41 & $-0,06$ \\
Estilo de vida & 6,19 & 0,7 & 0,137 \\
\hline
\end{tabular}

La dimensión consumo de alcohol, al igual que la de consumo de tabaco, expresó valores poco variables y sin un patrón coherente con la denominación de las agrupaciones. De hecho, a diferencia de lo esperado, el consumo de alcohol fue inverso a lo largo de los clústeres, desde muy saludable (3,6 DS 1,15), saludable (4,01 DS 1,08) y poco saludable (3.94 DS 1,14) (Tablas 4, 5 y 6).

La dimensión alimentación, al igual que la dimensión descanso y sueño y estrés, presentó variaciones pequeñas y con un patrón de mejora entre las agrupaciones. Los valores aumentaron desde poco saludable (1,44 DS 0,34), saludable $(1,6$ DS 0,33$)$ y muy saludable $(1,74$ DS 0,41$)$ (Tablas 4, 5 y 6).

Acorde con el comportamiento de la variación de los valores de cada dimensión a lo largo de las tres agrupaciones, puede observarse que en general las dimensiones de descanso y sueño, estrés y alimentación hacen contribuciones a la agrupación de los estudiantes en un mejor indicador, aunque el aumento es pequeño. Pero, de manera comparativa entre las agrupaciones, se observa que la mayor variación y aumento entre los mismos la expresó la actividad física, lo que es llamativo en términos de la contribución de cada dimensión en la conformación de los tres clústeres.

\section{DISCUSIÓN}

Los resultados del estudio reportan que las conductas de los estudiantes de Enfermería reflejan estilos de vida en su mayoría poco saludables, evidenciado por un importante consumo de alcohol, de tabaco y baja actividad física, información que se relaciona con lo mencionado por Bastías y Stiepovich ${ }^{(15)}$, donde en países como República Dominicana y Chile existe alta prevalencia al sedentarismo y por ello la adherencia a las actividades físicas es escasa.

Por otro lado, en países como Colombia y Portugal se reporta una preocupante situación en estudiantes de Enfermería por la alimentación con alto contenido en grasas y azúcar, así como la preferencia de comida chatarra, bajo consumo de frutas y verduras e incluso la omisión de las comidas $^{(16,17)}$. Este hábito influye directamente en la adquisición de comportamientos poco saludables 
que desarrollan con el tiempo enfermedades crónicas en la población adulto-joven.

La carga académica, el estrés y las preocupaciones del estudiante influyen en que los estilos de vida sean poco saludables, pues asumen conductas de riesgo como el consumo frecuente de alcohol y de tabaco, aumento en el consumo de cafeína, falta de descanso y sueño apropiados ${ }^{(16,18)}$. Un porcentaje importante de los estudiantes que participaron no está adoptando conductas saludables para la prevención de enfermedades crónicas, hallazgos que se relacionan con diferentes estudios donde los estudiantes no adoptan comportamientos de autocuidado adecuados debido a la carga académica, factores del entorno social, familiar, laboral y situaciones relacionadas con la economía y sustento diario ${ }^{(10,19)}$.

A pesar del panorama desfavorable respecto al estilo de vida poco saludable de los estudiantes, el estudio reporta una agrupación con estilo de vida saludable, siendo esta una importante parte de la población participante. Barragán et al. ${ }^{(20)}$ demostraron que estudiantes de diferentes carreras de una universidad en México poseen estilos de vida regular en un $66,40 \%$, bueno en un $15,80 \%$ y malo en un $17,80 \%$; aunque éste no sería el panorama ideal, demuestra que aún hay estudiantes que tienen conductas más saludables que otras que se podrían mejorar, como los hallazgos de esta investigación.

Algunos autores coinciden en que la nutrición, la actividad física, el alcohol, el tabaco y el estrés son indicadores determinantes a la hora de establecer los estilos de vida de las personas ${ }^{(16,18,21-}$ ${ }^{23)}$, situación que sustenta nuevamente los hallazgos del estudio respecto a las dimensiones establecidas para identificar el indicador de calidad de vida.

El panorama de los estilos de vida de los estudiantes de Enfermería y en general de los estudiantes universitarios, como lo muestran los estudios mencionados, hacen evidente que el indicador de estilo de vida propuesto está bien encaminado, pues las conductas de los estudiantes demuestran poco compromiso con el autocuidado y dificultad en el momento de adquirir estilos de vida sanos.

Es preocupante que estudiantes que se están preparando para brindar cuidado, atención y educación en salud a otra persona, tengan comportamientos que no reflejen lo que les enseñan a los pacientes. Rodríguez et al. ${ }^{(16)}$ refieren que una enfermera con sobrepeso no genera la misma confianza en el paciente que una enfermera de menor peso; Frank et al. ${ }^{(24)}$ señalan que un profesional que demuestra su estilo de vida saludable es más motivador al abordar a su paciente, por lo que tendría mayor éxito al mejorar las conductas de sus sujetos de cuidado.

Acorde con lo anterior, dado que muchas de las acciones de cuidado de enfermería tienen que ver con la promoción de estilos de vida saludables y el control de enfermedades crónicas no transmisibles, este estudio tiene implicaciones para la disciplina de enfermería, dentro de las que se debe considerar las pedagogías y didácticas en la enseñanza de los comportamientos sanos, el abordaje en la formación que trascienda a lo cognitivo e impacte en la conducta de los estudiantes, además de la reflexión de que la educación en salud y credibilidad del profesional ante el sujeto de cuidados depende en gran medida de los comportamientos saludables que éste puede evidenciar en el propio profesional ${ }^{(16)}$.

En este sentido, es evidente que el desarrollo del conocimiento disciplinar y la aplicación del mismo en la práctica del cuidado, no solo tiene que ver con el conocer, sino también con el ser y el hacer. Desde esta perspectiva, se requiere que desde la formación, las universidades y en particular las facultades de Enfermería, se conviertan en comunidades de cuidado, que propicien espacios idóneos para la promoción y mantenimiento de estilos de vida saludable ${ }^{(24)}$.

El indicador de estilo de vida refleja los comportamientos de los estudiantes universitarios como se reporta en la literatura; el ambiente académico y las situaciones de carácter psicosocial, son factores influyentes en la adopción de rutinas y conductas saludables o no saludables; se evidencia que si bien hay estudiantes con estilos de vida saludables, el número de jóvenes con comportamientos mal sanos es importante ${ }^{(10,19,25,26)}$.

Esta situación sugiere la necesidad de políticas universitarias que ayuden a mejorar los estilos de vida de los estudiantes universitarios; algunas universidades en Latinoamérica han implementado programasqueimpulsan ala comunidad universitaria a cambiar ciertos hábitos de vida. En Colombia se conformó la Red Colombiana de Instituciones de Educación Superior y Universidades Promotoras de la Salud (REDCUPS) y en Chile la Red 
Universitaria de Promoción y Autocuidado (RED UPRA), entidades que desarrollan actividades o investigación relacionadas con las necesidades, en materia de salud, de los estudiantes $(27,28)$. En México, la Universidad Autónoma de Zacatecas creó un programa con el objetivo de impulsar a la comunidad a la responsabilidad individual referente a los estilos de vida saludables, generando propuestas en torno a la actividad física, nutrición, salud sexual, salud mental, entre otros ${ }^{(28)}$.

A pesar de que existen programas que promueven mejorar los estilos de vida de los estudiantes universitarios, los resultados en la modificación de estilos de vida saludables no han sido óptimos ${ }^{(27,28)}$, dado que se necesita implementar intervenciones que cubran las necesidades individuales de los estudiantes y que se realicen desde el momento en que el estudiante ingresa a la universidad. Además, se requiere conocer las variables que intervienen en el desarrollo y adherencia a estilos de vida saludables en cada entorno particular, pues es claro que no solo se relacionan con lo cognitivo, sino con una compleja interacción de factores psicológicos y sociales. Por ello es necesario realizar estudios cualitativos que permitan conocer las realidades particulares con profundidad y a la vez otorguen la información suficiente para diseñar intervenciones a la medida.

\section{CONCLUSIONES}

Los estudiantes de Enfermería de la Universidad Autónoma del Estado de México se agrupan principalmente en el indicador de estilo de vida saludable; sin embargo, un número considerable

\section{REFERENCIAS}

1. Grimaldo MP. Estilo de vida saludable en estudiantes de posgrado de Ciencias de la Salud. Psicología y salud. [Internet]. 2012 Ene-Jun. [citado 03 Jun 18]; 22(1):75-87. Disponible en: http:// psicologiaysalud.uv.mx/index.php/psicysalud/ article/view/559

2. Guzmán AJ, Salcedo LA, Melgarejo VM. Caracterización de los estilos de vida saludables en estudiantes de licenciatura en educación básica de la facultad de estudios a distancia CREAD- de personas poseen estilos de vida poco saludables que pueden influir en la aparición de enfermedades crónicas si no se mejoran los hábitos y costumbres a tiempo. Los resultados del estudio muestran que prácticas como una inadecuada dieta, consumo regular de tabaco y/o alcohol, falta de actividad física y frecuente sedentarismo, conducen a estilos de vida poco saludables; conforme cambien estas prácticas, mejorará el estilo de vida y disminuirá la prevalencia a padecer una ECNT.

El resultado de las agrupaciones evidenció que las dimensiones de estrés, consumo de tabaco y consumo de alcohol reportan alta puntuación en el indicador de estilo de vida, aun cuando este sea "muy saludable", pues estos comportamientos malsanos son constantes en los estudiantes; así como la dimensión descanso y sueño que evidencia, para las tres agrupaciones, que los estudiantes no están descansando lo suficiente.

El estudio evidenció que ser estudiantes de carreras que promueven los estilos de vida saludables a sus sujetos de cuidado, no significa que adopten comportamientos sanos en su desarrollo personal. De esta manera, el indicador de estilo de vida propuesto fue apropiado para los estudiantes de Enfermería, pues es el reflejo de las conductas sanas y malsanas que ejercen durante su rol académico, lo que sugiere, para futuras investigaciones, realizar estudios que permitan describir los factores que influyen en las conductas malsanas de los estudiantes, el desarrollo de intervenciones y políticas que mejoren sus estilos de vida.

Como limitación del estudio se señala el tipo de muestreo, ya que se desarrolló un muestreo de tipo intencional.

Duitama. Rev salud hist sanid [Internet]. 2016 JulDic. [citado 17 Jun 18]; 11(2):3-28. Disponible en http://agenf.org/ojs/index.php/shs/article/view/158

3. Lema L, Salazar I, Varela M, Tamayo J, Rubio A, Botero A. Comportamiento y salud de los jóvenes universitarios: satisfacción con el estilo de vida. Pensam. Psicol. 2009; 5(12): 71-88.

4. Organización Mundial de la Salud. Enfermedades no transmisibles [Internet]. Ginebra: OMS; 2018 [citado 20 Jun 2018]. Disponible en: https:// www.who.int/es/news-room/fact-sheets/detail/ noncommunicable-diseases 
5. Lumbreras I, Moctezuma MG, Dosamantes LD, Medina MA, Cervantes M, López M del RR, et al. "Estilo de vida y riesgos para la salud en estudiantes universitarios: hallazgos para la prevención". Revista Digital Universitaria [Internet]. 2009 Feb 10. [citado 30 Jul 2018]; 10(2):1-14. Disponible en: http://www.revista.unam.mx/vol.10/num2/ art12/int 12. htm

6. Páez ML, Castańo JJ. Estilos de vida y salud en estudiantes de una facultad de psicología. Psicol. caribe. [Internet]. 2010 Ene-Jun. [citado 30 Jul 2018]; (25):155-178. Disponible en: http://www.scielo.org.co/scielo.php?script=sci arttext\&pid=S0123-417X2010000100008

7. Coral R del C, Vargas LD. Estrategias que promueven estilos de vida saludables. rev. udcaactual.divulg.cient. [Internet]. 2014 Ene-Jun. [citado 30 Jul 2018]; 17(1):35-43. Disponible en: http://www.scielo.org.co/scielo.php?pid=S0123$42262014000100005 \& \mathrm{script}=\mathrm{sci}$. abstract\&tlng=es

8. Tirodimos I, Georgouvia I, Savvala TN, Karanika E, Noukari D. Healthy lifestyle habits among Greek university students: differences by sex and faculty of study. East Mediterr Health J [Internet]. 2009 May-Jun. [cited 20 Jul 2018];15(3): 722-728. Available from: http://apps.who.int/iris/bitstream/ handle/10665/117691/15_3_2009_0722_0728. pdf? sequence $=1$ \&isAllowed $=y$

9. Reza CG, Ferreira MA, Silva RC, Gandarilla JV, Solano GS, Martínez VG. Profile of Mexican students in nursing clinics. Esc Anna Nery. 2016; 20(1):11-16.

10. Laguado Jaimes E, Gómez Díaz MP. Estilos de vida saludable en estudiantes de Enfermería en la Universidad Cooperativa de Colombia. Hacia promoc. Salud. [Internet]. 2014 Ene-Jun. [citado 01 Jun 18]; 19(1):68-83. Disponible en: http:// www.scielo.org.co/pdf/hpsal/v19n1/v19n1a06.pdf

11. Saad Acosta C, Ibáńez E, León C, Colmenares C, Vega N, Díaz Y. Cambios en los hábitos alimentarios de los estudiantes de enfermería de la Universidad El Bosque durante su proceso de formación académica, Bogotá, D.C., 2007. Rev Colomb Enfermería [Internet]. 2008 [citado 30 May 2018]; 3(3): 51-60. Disponible en: http://m.uelbosque. edu.co/sites/default/files/publicaciones/revistas/ revista_colombiana_enfenferme/volumen3/ cambios_habitos_alimentarios_estudiantes_ enfermeria_universidad_el_bosque.pdf

12. Arguello M, Bautista Y, Carvajal J, De Castro K, Díaz D, Escobar M, et al. Estilos de vida en estudiantes del área de la salud de Bucaramanga. Rev Psicol Univ Antioquia. [Internet]. 2009 JulDic. [citado 7 May 18]; 1(2):27-41. Disponible en: https://aprendeenlinea.udea.edu.co/revistas/index. php/psicologia/article/view/10019

13. Bennasar M. Estilos de vida y salud en estudiantes universitarios: la universidad como entorno promotor de la salud. [Tesis Doctoral]. Islas Baleares (España): Universidad de las Islas Baleares. Departamento de Enfermería y Fisioterapia; 2012. $362 \mathrm{p}$.

14. Johnson RA, Wichern DW. Applied Multivariate Statistical Analysis. 6th ed. New York: Pearson Education Inc; 2007. 808 p.

15. Bastías Arriagada EM, Stiepovich Bertoni J. Una revisión de los estilos de vida de estudiantes universitarios Iberoamericanos. Cienc. enferm. 2014; 20(2):93-101.

16. Rodríguez-Gázquez MA, Chaparro-Hernández $S$, González-López JR. Lifestyles of nursing students from a Colombian public university. Invest Educ Enferm [Internet]. 2016 Apr [cited 2018 Sept 5]; 34(1):94-103. Available from: https://www.ncbi. nlm.nih.gov/pubmed/28569978

17. Ferreira FMPB, Mota LAN, Brito IS, Santos MR. Perfil de saúde dos estudantes de enfermagem: diagnóstico epidemiológico a partir do modelo PRECEDE-PROCEED. Rev Enf. Ref. 2017; 4(15):91-100.

18. Lehmann F, Von Lindeman K, Klewer J, Kugler J. BMI, physical inactivity, cigarette and alcohol consumption in female nursing students: a 5-year comparison. BMC Med Educ [Internet]. 2014 Apr [cited 2018 Sep 5];14(1):82. Available from: http://bmcmededuc.biomedcentral.com/ articles/10.1186/1472-6920-14-82

19. Sánchez-Ojeda MA, De Luna-Bertos E. Hábitos de vida saludable en la población universitaria. Nutr Hosp. 2015; 31(5):1910-9.

20. Barragán-Ledesma L, González-Preza MG, EstradaMartínez S, Hernández-Cosain Y, HernándezCosain E, Ríos-Valles J, et al. Estilo de vida y dimensiones, en estudiantes universitarios de área de la salud. Cienc. humanismo salud. [Internet]. 2015 May-Ago. [citado 5 Sept 2018]; 2(2):5363. Disponible en: http://revista.medicina.uady. $\mathrm{mx} / \mathrm{revista} / \mathrm{index} . \mathrm{php} / \mathrm{cienciayhumanismo/article/}$ view/36

21. Novoa-Sandoval P, Pérez-Acuña K, AchataEspinoza M, Toro-Huamanchumo CJ. Síndrome de intestino irritable en estudiantes de medicina de Latinoamérica: ¿un problema pasado por alto? Rev. gastroenterol. Perú. 2017; 37(2):190-1.

22. Moreno-Bayona JA. Niveles de sedentarismo en estudiantes universitarios de pregrado de Colombia. Rev Cubana Salud Pública [Internet]. 2018 Jul-Sept. [citado 5 Sept 2018]; 44(3): 553-566. Disponible en: http://scielo.sld.cu/scielo.php?script=sci_ 
arttext\&pid=S0864-34662018000300009

23. Rodríguez-Gázquez M, Chaparro-Hernández S, González-López JR. Are first-year nursing students' lifestyles coherent with their future career? Int J Nurs Pract [Internet]. 2017 Jan 23 [cited 5 Sep 2018]; 23(2):e12511. Available from: http://doi. wiley.com/10.1111/ijn.12511

24. Frank E, Breyan J, Elon L. Physician disclosure of healthy personal behaviors improves credibility and ability to motivate. Arch Fam Med. 2000; 9(3):287-290.

25. Padilla-García CI, Jaimes-Valencia ML, FajardoNates S, Ramos-Franco AM. Factores de riesgo cardiovascular y estilos de vida de estudiantes universitarios. Med Unab. [Internet]. 2014 Ago-Nov. [citado 12 Mar 2019]; 17(2):81-90. Disponible en: https://revistas.unab.edu.co/index. php/medunab/article/view/2171
26. Mu M, Uribe A. Estilos de vida en estudiantes universitarios. Psicogente [Internet]. 2013 [citado 12 Mar 2019]; 16(30):356- 67. Disponible en: http:// revistas.unisimon.edu.co/index.php/psicogente/ article/view/1923

27. Organización Panamericana de la Salud. Una Nueva Mirada al Movimiento de Universidades Promotoras de la Salud en las Américas. [Internet]. 2009; Pamplona (España): IV Congreso Internacional de Universidades Promotoras de la Salud, Universidad Pública de Navarra; [citado 5 Sep 2018]. 37 p. Disponible en: https://www.paho. org/hq/dmdocuments/2012/2009-ArroyoRiceMiradaMovimientoUPSenAmericas.pdf

28. Heraud SB. Universidades saludables: una apuesta a una formación integral del estudiante. Revista de Psicología. 2013; 31(2):287-314. 